\title{
Liquid Viruses by Nanoscale Engineering of Capsid Surfaces
}

\author{
Avinash J. Patil, Nina McGrath, J. Elaine Barclay, David J. Evans, Helmut Cölfen, \\ Ian Manners, Adam W. Perriman, and Stephen Mann*
}

\begin{abstract}
Amongst a wide variety of possible biomolecular components for integration into nanotechnological systems, bacteriophages ${ }^{[1]}$ and plant viruses ${ }^{[2]}$ are of special interest because of their intrinsic monodispersity, robust and highly uniform architectures, and spatially defined surface chemistry. Virus particles have been used as templates for the nucleation and growth of inorganic nanomaterials, ${ }^{[3,4]}$ compartments for encapsidation of nanoparticles,,$^{[5,6]}$ lyotropic liquid crystals for the fabrication of organized mesostructured materials and functional devices, $, 7,8]$ addressable surfaces for chemical functionalization, ${ }^{[9]}$ and delivery vehicles for vaccines and drugs. ${ }^{[10-12]}$ In particular, cowpea mosaic virus (CPMV), which is a $28 \mathrm{~nm}$-diameter plant virus with icosohedral geometry, has been used for a wide range of applications, for example in microelectronics, ${ }^{[13]}$ biomineralisation, ${ }^{[14]}$ nanoscale array construction, ${ }^{[15]}$ and epitope display in potential plant vaccines, ${ }^{[16,17]}$ and empty CPMV-like particles devoid of their ssRNA core have been exploited for entrapping metal and metal oxide nanoparticles. ${ }^{[18,19]}$ Whilst the above examples clearly highlight the potential of using viruses in nanotechnology, the processing and engineering of these biological components is currently limited to methods based primarily on aqueous media due to the insolubility and potential destabilization of the self-assembled viroid structure in organic solvents, and general difficulty and safety concerns associated with storage and manipulation of these biological materials as freezedried powders. Indeed, like nanoscale objects in general, ${ }^{[20]}$ the
\end{abstract}

Dr. A. J. Patil, ${ }^{[+]}$N. McGrath, ${ }^{[+]}$Dr. A. W. Perriman,

Prof. S. Mann

Centre for Organized Matter Chemistry

School of Chemistry

University of Bristol

Bristol BS8 1TS, UK

E-mail: s.mann@bristol.ac.uk

J. E. Barclay, Prof. D. J. Evans

Department of Biological Chemistry

John Innes Centre

Norwich Research Park

Colney, Norwich, NR4 7UH UK

Prof. H. Cölfen

Physikalische Chemie

Universität Konstanz

Universitätsstr. 10, D-78457 Konstanz, Germany

Prof. I. Manners

School of Chemistry

University of Bristol

Bristol BS8 1TS, UK

$[+]$ These authors contributed equally to this work. phase behaviour of viruses is constrained because the dimensions of the capsids are greater than the range of their attractive intermolecular force fields, and this disparity results in the absence of the liquid phase devoid of solvent. As a consequence, there are no known examples of pure liquid viruses.

The ability to prepare solvent-free liquids comprising extremely high concentrations of structurally and functionally intact virus nanoparticles should have significant impact in advancing the bioinspired design and processing of biologically derived nanostructures. Accessing the pure liquid state will depend on the size and shape of the capsids, and the strength and range of their intermolecular attractive forces. ${ }^{[21]}$ In this regard, recent studies with ferritin ${ }^{[22]}$ and myoglobin ${ }^{[23]}$ have shown that judicious surface engineering of the globular architectures of these proteins produces a solvent-free liquid state at close to room temperature. The liquids are in the form of thermally stable viscoelastic melts with close to zero vapour pressure, and comprise intact ferritin or myoglobin molecules that exhibit non-Newtonian/Newtonian behaviour or reversible dioxygen binding properties, respectively. Extending this approach to spherical virions such as CPMV is challenging because the molecular dimensions are significantly larger than those of myoglobin (ca. $4 \mathrm{~nm}$ ) and ferritin (12 nm), and as a consequence engineering the concomitant modification and increase in the range of the attractive intermolecular force is potentially more difficult. Nevertheless, here we demonstrate a synthetic strategy that accesses the liquid phase of a virus in the absence of solvent, and show that these concentrated viscoelastic fluids are highly effective in plant infectivity. Moreover, the liquid virus transforms into a soft solid below $28{ }^{\circ} \mathrm{C}$, and can be dissolved in aqueous solutions or a range of organic solvents, suggesting that these bionanomaterials could also be used as novel storage and transport media, and exploited for the development of non-aqueous processing routes in virusbased nanotechnology.

The following strategy was used to surface engineer CPMV capsids to produce a solvent-free liquid virus (see Supporting Information). Wild-type CPMV (WT-CPMV) consists of 60 copies of each of 2 different coat proteins comprising large (L) or small (S) subunits. As the combined amino acid sequences of the two different CPMV coat proteins contain 51 acidic acid residues (21 glutamic acid and 30 aspartic acid), we reasoned that this high anionic charge density could be exploited for surface engineering to produce stoichiometric constructs capable of undergoing thermally induced melting in the absence of water. To initiate this strategy, we cationized the WT-CPMV capsids in aqueous solution by carbodiimide-mediated covalent coupling of ethylenediamine (EDA) to surface-accessible 


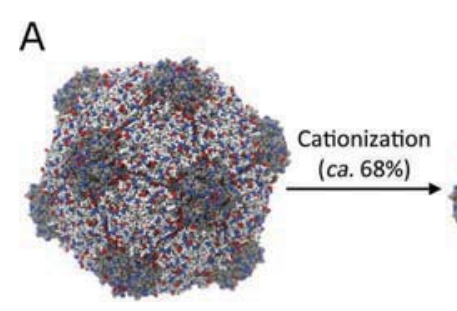

I
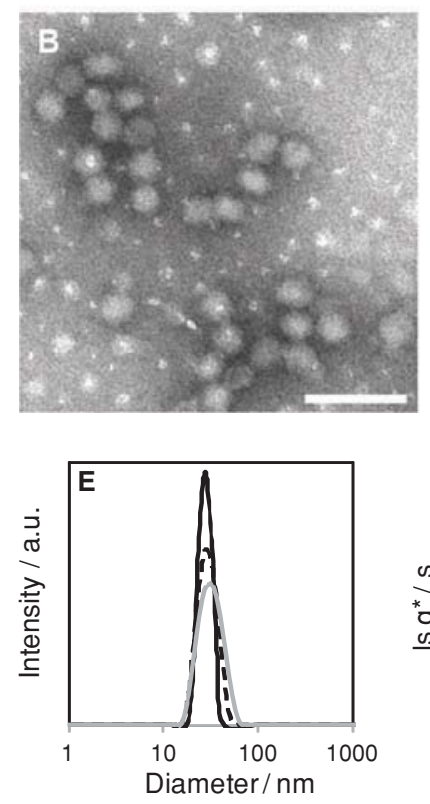

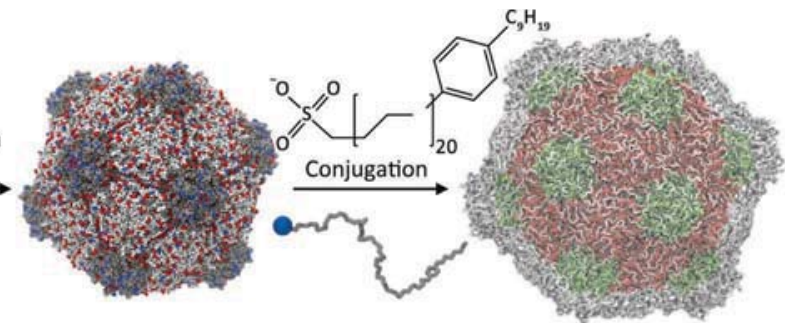

II
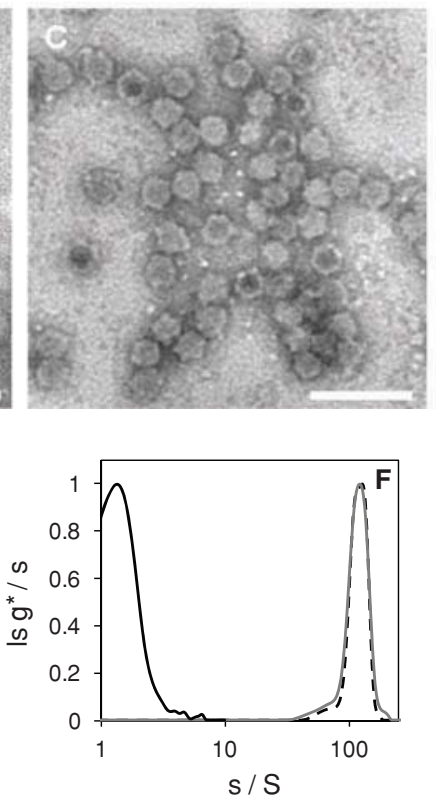

III
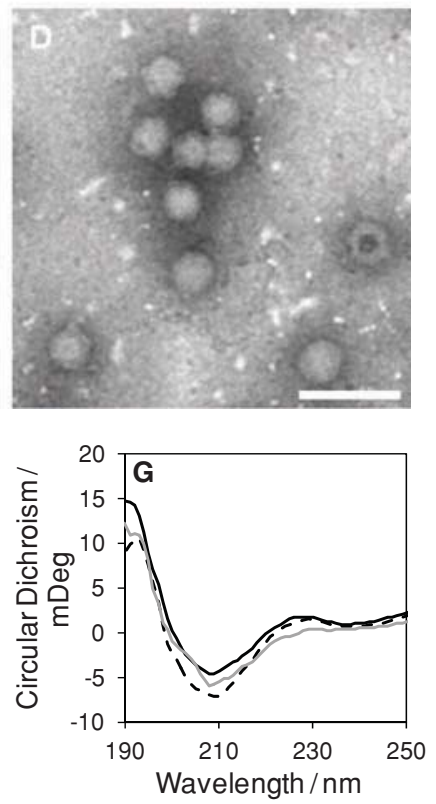

Figure 1. Synthetic strategy used in the surface engineering of CPMV for the fabrication of a solvent-free liquid virus. (A) Schematic illustration showing a molecular model of an icosohedral WT-CPMV particle decorated with acidic (blue) and basic (red) residues (I). Surface functionalization via carbodiimide-mediated covalent coupling of EDA to surface accessible acidic residues produces a cationized-CPMV capsid [Cat-CPMV] (II). The [Cat-CPMV] conjugate is then electrostatically complexed with an anionic polymer-surfactant $\mathbf{S}$ to produce an ionic construct [Cat-CPMV][S] in aqueous solution (III). (B-D) Transmission electron micrographs of WT-CPMV (B), Cat-CPMV (C) and [Cat-CPMV][S] (D) prepared by drop-casting from aqueous solutions, showing intact virus particles with an average diameter of 27, 28 and $36 \mathrm{~nm}$ respectively. Samples were stained with $1 \%$ phosphotungstic acid prior to imaging. Scale bars $=200 \mathrm{~nm}$. (E) DLS intensity plots of aqueous suspensions of WT-CPMV (dashed line), Cat-CPMV (black line) and [Cat-CPMV][S] (grey line) particles, showing uni-modal distributions with mean hydrodynamic diameters of 30, 29 and $33 \mathrm{~nm}$, respectively. (F) Sedimentation coefficient distributions for $\mathbf{S}$ alone (black line), Cat-CPMV (grey line) and [Cat-CPMV][S] conjugate (dashed line) run in $10 \mathrm{mM}$ phosphate buffer solution (PBS) at $25^{\circ} \mathrm{C}$. No evidence of unbound $\mathbf{S}$ was observed in the [Cat-CPMV][S] samples. (G) Room temperature far-UV CD spectra of aqueous solutions (ca. $0.1 \mathrm{mg} \mathrm{mL}^{-1}$ ) of WT-CPMV (dashed line), Cat-CPMV (black line) and [Cat-CPMV][S] conjugate (grey line); in each case peaks at 195 and $210 \mathrm{~nm}$ are observed.

acidic side chains (Figure 1A). This resulted in reversal of the net surface charge from a zeta potential value of $-12 \mathrm{mV}$ for an aqueous dispersion of WT-CPMV to $+14.7 \mathrm{mV}$ for the cationized CPMV conjugate (Cat-CPMV).

Matrix-assisted laser desorption/ionisation-time of flight (MALDI-TOF) mass spectroscopy gave a cationization efficiency of $\mathrm{ca}$. $68 \%$, corresponding to the modification of 35 acidic amino acid residues per subunit pair-that is, 2100 EDA molecules per virion (Supporting Information Figure S1). This value was higher than previous reports on the number of accessible sites on the capsid outer surface, ${ }^{[24,25]}$ and higher than the number of acidic amino acid residues residing on the outer surface of the virion, suggesting that EDA penetrated inside the capsid and reacted with acidic amino acid residues present on the internal surface. Combining the accessible basic and EDA-modified residues on the outer surface equated to a total of 42 cationic sites per subunit pair, equivalent to 2520 positively charged surface groups per virion. Transmission electron microscopy (TEM) images of WT-CPMV or Cat-CPMV showed in both cases discrete 28-nm diameter spherical nanoparticles, indicating that the virions remained structurally intact and nonaggregated after cationization (Figure 1B \& 1C).

As a second step towards the preparation of a solvent-free liquid of CPMV particles, we exploited the Cat-CPMV conjugate as a polycationic platform for the electrostatically directed assembly of a stoichiometrically coupled polymersurfactant coronal layer. This was achieved by addition of the anionic polymer-surfactant, poly(ethylene glycol) 4-nonylphenyl 
3-sulfopropyl ether $\left(\mathrm{C}_{9} \mathrm{H}_{19}-\mathrm{C}_{6} \mathrm{H}_{4}-\left(\mathrm{OCH}_{2} \mathrm{CH}_{2}\right)_{20} \mathrm{O}\left(\mathrm{CH}_{2}\right)_{3} \mathrm{SO}_{3}^{-}\right.$ (S)) (Figure 1A) to an aqueous solution of Cat-CPMV, followed by extensive dialysis to ensure complete removal of unbound $\mathbf{S}$ molecules. Corresponding TEM micrographs of the polymersurfactant/virus ([Cat-CPMV][S]) construct showed spherioidal capsids with a mean particle diameter of $36 \mathrm{~nm}$ (Figure 1D), indicating that the coronal shell was assembled without loss of the capsid architecture. Dynamic light scattering (DLS) measurements of an aqueous dispersion of [Cat-CPMV][S] gave a hydrodynamic diameter of $33 \mathrm{~nm}$ that was $3 \mathrm{~nm}$ larger than the corresponding values for Cat-CPMV and WT-CPMV (Figure $1 \mathrm{E})$, and which was consistent with the assembly of a dense, nano-thin layer of $\mathbf{S}$ molecules on the surface of the cationized virus particles. The presence of a coronal layer was also confirmed by zeta potential measurements that gave a value of -7 $\mathrm{mV}$ for the aqueous [Cat-CPMV][S] construct compared with $+14.7 \mathrm{mV}$ for [Cat-CPMV], and by gel electrophoresis, which revealed greatly reduced mobility compared to the WT-CPMV control (Supporting Information Figure S2A). UV-Vis spectra showed an absorbance peak at $275 \mathrm{~nm}$, corresponding to the $\pi \rightarrow \pi *$ transition of the phenyl moiety of the polymer-surfactant chain associated with [Cat-CPMV][S] (Supporting Information Figure S2B). Sedimentation coefficient distributions obtained by analytical ultracentrifugation (AUC) showed only a single band at $123.5 \mathrm{~S}$ (Figure $1 \mathrm{~F}$ ) that corresponded to a discrete nanoscale component associated with aqueous [Cat-CPMV][S]; significantly, no evidence of unbound $\mathbf{S}$ was observed. The same sedimentation coefficient was obtained for WT-CPMV and CatCPMV (Supporting Information Figure S2C), indicating that the increase in molar mass of the polymer-surfactant/CPMV construct was offset by a reduction in density associated with formation of the coronal layer. A binding stoichiometry of 1 : 3417 polymer-surfactant molecules per CPMV virion was calculated from the measured sedimentation distributions, particle diameters and molar masses (densities) using calculated frictional coefficients (see Supporting Information). This corresponded to a polymer-surfactant content of $52 \mathrm{wt} \%$, equivalent to a grafting density of one $\mathrm{S}$ molecule per $0.72 \mathrm{~nm}^{2}$. Given this high grafting density, we used circular dichroism spectroscopy to determine the influence of the surface modifications on the secondary structure of the coat protein subunits. The spectra for aqueous dispersions of WT-CPMV, Cat-CPMV and [Cat-CPMV] [S] were almost identical (Figure 1G), and showed peaks centred around 195 and $210 \mathrm{~nm}$ that corresponded to a predominately $\beta$-sheet structure, ${ }^{[26]}$ indicating that the native secondary structure was retained during the various surface engineering procedures.

The final step towards accessing a solvent-free liquid state of the virus entailed exhaustive lyophilization of aqueous solutions of the single-component [Cat-CPMV][S] nanoconstruct to produce a low-density dehydrated white solid. Remarkably, upon thermal annealing, the freeze-dried solid melted into a viscous translucent fluid with a CPMV concentration of $c a .475 \mathrm{mg} \mathrm{mL}^{-1}$ (Figure 2A-C). Subsequent cooling of the sample to room temperature gave a soft, colourless, translucent solid. Differential scanning calorimetry (DSC) performed on the lyophilized [Cat-CPMV][S] construct showed a solid to liquid melting transition at $28{ }^{\circ} \mathrm{C}$, as well as a solid-state crystallisation transition at $-30{ }^{\circ} \mathrm{C}$ (Figure 2D). The former was close to the melting point observed for pure $\mathbf{S}$, suggesting that long range intermolecular attractive interactions between the polymer-surfactant coronal layers of the dehydrated [Cat-CPMV][S] construct were responsible for the onset of the liquid state. Significantly, thermogravimetric analysis (TGA) of the [Cat-CPMV][S] gave a water content of $0.6 \%$ (Figure $2 \mathrm{E}$ ), which corresponded to approximately 46 water molecules per polymer-surfactant-conjugated subunit pair, which was considerably less than the number of amino acid sites known to strongly bind water (123 water molecules per subunit pair, $3.3 \mathrm{wt} \%),{ }^{[27]}$ and an order of magnitude lower than that required for protein motion and function (7\%). ${ }^{[28]}$

As a step towards assessing the potential exploitation of the liquid virus phase as a novel biomaterial fluid, we undertook a series of experiments to determine the physical, structural and functional properties of the $[$ Cat-CPMV] $[\mathbf{S}]$ virions. The rheological behaviour of the [Cat-CPMV][S] melt was determined using oscillation measurements performed at $40^{\circ} \mathrm{C}$. Amplitude sweeps indicated that under these conditions the melt was a viscoelastic fluid (Supporting Information Figure S3). Reproducible frequency sweeps performed at $1 \mathrm{~Hz}$ and $40{ }^{\circ} \mathrm{C}$ in the viscoelastic region showed storage $\left(\mathrm{G}^{\prime}\right)$ and loss $\left(\mathrm{G}^{/ /}\right)$moduli with values of 2.73 and $2.98 \mathrm{KPa}$, respectively. A relaxation time of approximately $0.6 \mathrm{~s}$ was calculated from the cross-over point of $\mathrm{G}^{\prime}$ and $\mathrm{G}^{/ /}$(Figure $2 \mathrm{~F}$ ), indicating that the melt exhibited solid-like behaviour over short time periods, yet flowed with liquid-like behaviour over longer time intervals. As the theoretical volume fraction of CPMV particles in the [Cat-CPMV] [S] solvent-free liquid was 0.47 , these flow properties were consistent with classical models of colloidal suspensions of hard monodisperse spherical particles, which predict that the onset of solid-like crystallization from the fluid phase occurs above a particle volume fraction of $0.49 .{ }^{[29]}$

Given the high level of molecular crowding, we used attenuated total reflectance-Fourier transform infrared (ATR-FTIR) spectroscopy to assess the preservation of coat protein secondary structure in the [Cat-CPMV][S] melt. The spectra exhibited protein amide I and II bands centred around $1630 \mathrm{~cm}^{-1}$ and $1523 \mathrm{~cm}^{-1}$, respectively, as well as peaks corresponding to $\mathrm{C}-\mathrm{C}$ bond stretches of the para-substituted phenyl ring of S at 1612, 1584 and $1512 \mathrm{~cm}^{-1}$ (Figure 2G). The amide I and II bands were essentially unchanged from WT-CPMV, indicating that the predominantly $\beta$-sheet secondary structure of the coat proteins was retained in the liquid state devoid of water. A broad shoulder peak at $1065 \mathrm{~cm}^{-1}$, corresponding to the C-O stretching frequency from the RNA backbone ${ }^{[30]}$ was also present in the spectrum of the [Cat-CPMV][S] melt, as well as in the lyophilized WT-CPMV sample (Supporting Information Figure S4). Although partially masked by the high intensity of the C-O stretch from the polyethylene glycol (PEG) moiety of $\mathbf{S}$, the data signified that surface modification, dehydration and melting did not remove the RNA from the virus capsids. Significantly, the [Cat-CPMV][S] melt could be readily dissolved in water to produce aqueous dispersions of the intact polymer-surfactant/virus construct without loss of structure or RNA content. Moreover, temperature-dependent DLS measurements indicated that the thermal stability of the re-dissolved [Cat-CPMV][S] construct was considerably increased compared with aqueous dispersions of WT-CPMV (Supporting Information Figure S5). Interestingly, increasing the ionic strength-for 

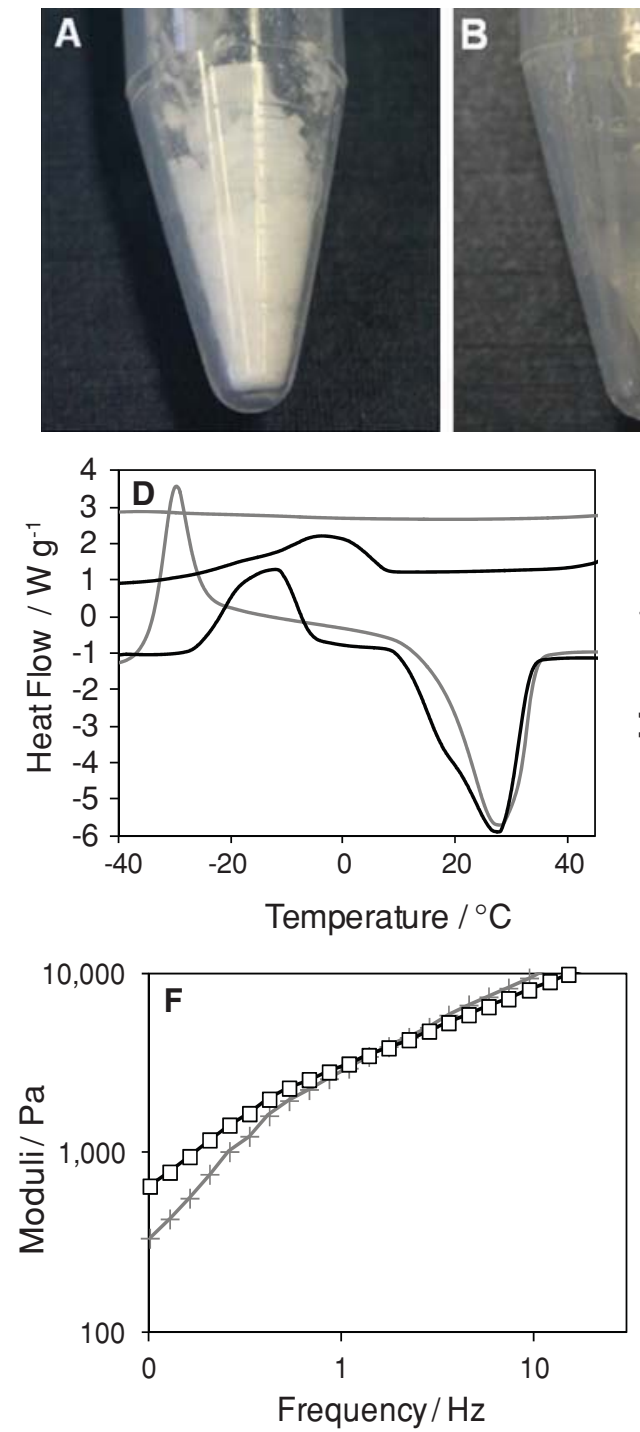
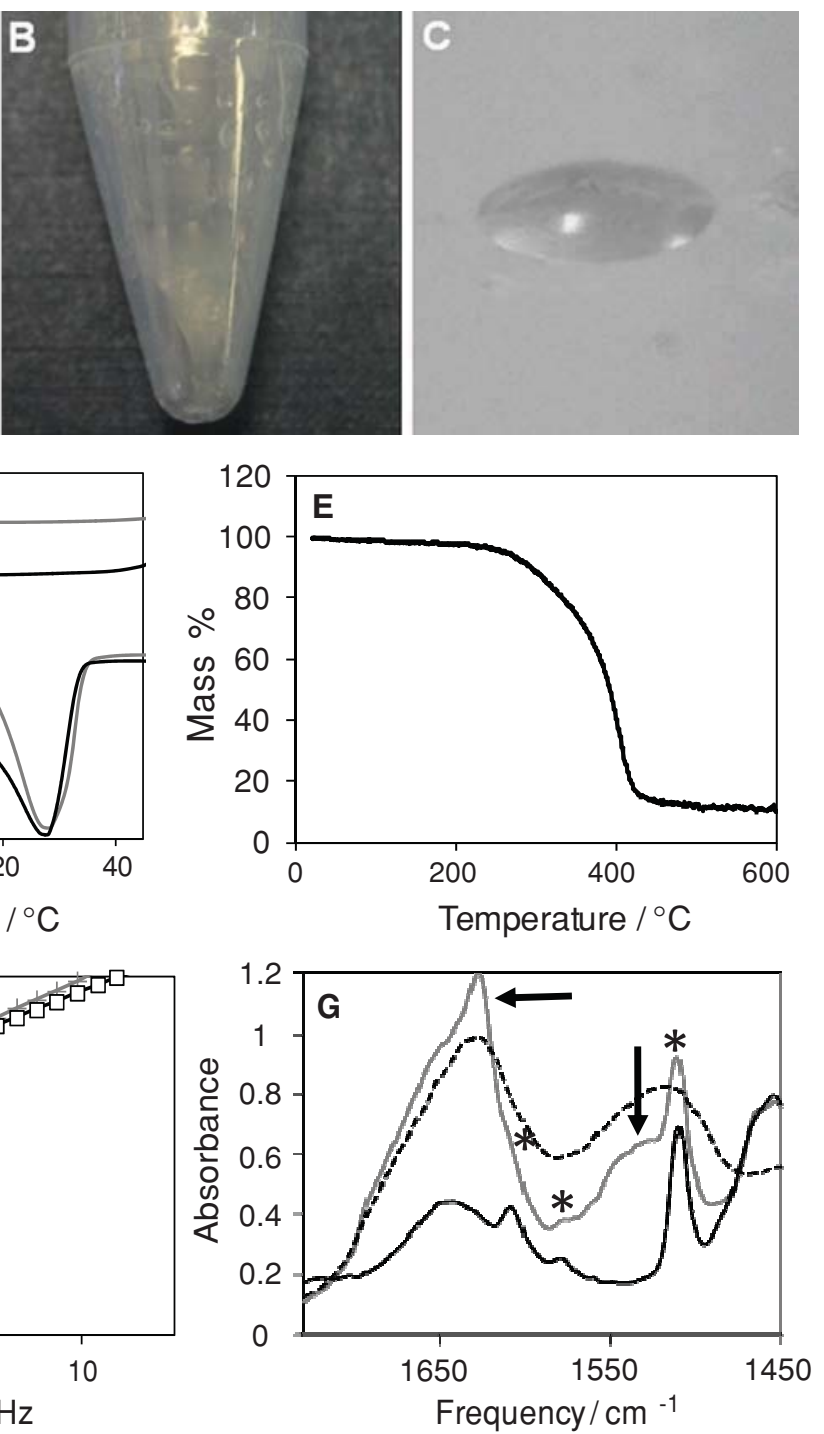

Figure 2. (A, B) Optical photographs of sample tubes containing [Cat-CPMV][S] showing a low density white solid obtained after lyophilization (A), and a highly viscous translucent fluid obtained after annealing the freeze-dried material at $65{ }^{\circ} \mathrm{C}$ for 10 minutes (B). (C) Optical image showing single liquid droplet of a solvent-free [Cat-CPMV][S] melt placed on a glass side at $65^{\circ} \mathrm{C}$. (D) DSC thermogram of a [Cat-CPMV][S] melt (grey line) and pure $\mathbf{S}$ (black line), showing melting transitions at 27.5 and $28^{\circ} \mathrm{C}$, respectively. DSC experiments were performed at an annealing rate of $10{ }^{\circ} \mathrm{C}$ min ${ }^{-1}$. (E) TCA profile of a [Cat-CPMV][S] melt showing minimal weight loss below $200{ }^{\circ} \mathrm{C}$, and a decomposition temperature of $402{ }^{\circ} \mathrm{C}$. The temperature ramp-rate was $10^{\circ} \mathrm{C} \mathrm{min}^{-1}$ and the sample was held at $110^{\circ} \mathrm{C}$ for 60 minutes. (F) Storage $(\mathrm{G} /,+$ ) and loss (G/l, +) moduli of a [Cat-CPMV][S] melt as a function of frequency. Measurements were performed at $40{ }^{\circ} \mathrm{C}$ at a shear stress of $60 \mathrm{~Pa}$, in the viscoelastic region of the material (see amplitude sweeps in Figure S3). (G) ATR-FTIR spectra of WT-CPMV (upper black line), [Cat-CPMV][S] melt (grey line) and $\mathbf{S}$ (lower black line). Arrows show amide I (1630 $\mathrm{cm}^{-1}$ ) and II $\left(1523 \mathrm{~cm}^{-1}\right)$ bands for the coat proteins, and C-C bond stretches of the para-substituted ring of $\mathbf{S}$ are highlighted (*).

example in the presence of $500 \mathrm{mM} \mathrm{NaCl-resulted} \mathrm{in} \mathrm{partial}$ dissociation of the $\mathbf{S}$ molecules to produce a mixture of dispersed [Cat-CPMV] and [Cat-CPMV][S] virions (Supporting Information Figure S6).

In light of the above observations, we reasoned that the [Cat-CPMV][S] melt could serve as a highly concentrated agent for use in plant infectivity provided that the polymersurfactant coronal layer did not constrain host processing of the viral RNA. We tested this by undertaking a series of infection studies that assessed the activity of the various constructs with respect to leaves of the plant, V. unguiculata. Plant leaves infected with aqueous solutions of WT-CPMV, Cat-CPMV or
[Cat-CPMV][S] conjugates exhibited typical visual symptoms of infection (Figure 3A-E). The yields of virions produced in the presence of Cat-CPMV or [Cat-CPMV][S] corresponded to 80 and $82 \mathrm{wt} \%$, respectively, of the virion yield obtained with WT-CPMV. Significantly, direct application of the solvent-free liquid [Cat-CPMV][S] to the leaf surface also resulted in successful infectivity (Figure 3D). In each case, gel electrophoresis of the replication products isolated after infection showed mobilities consistent with WT-CPMV (Figure 3F), indicating that the viral RNA contained within the surface engineered capsids remained sufficiently intact and accessible to host cell processing. 

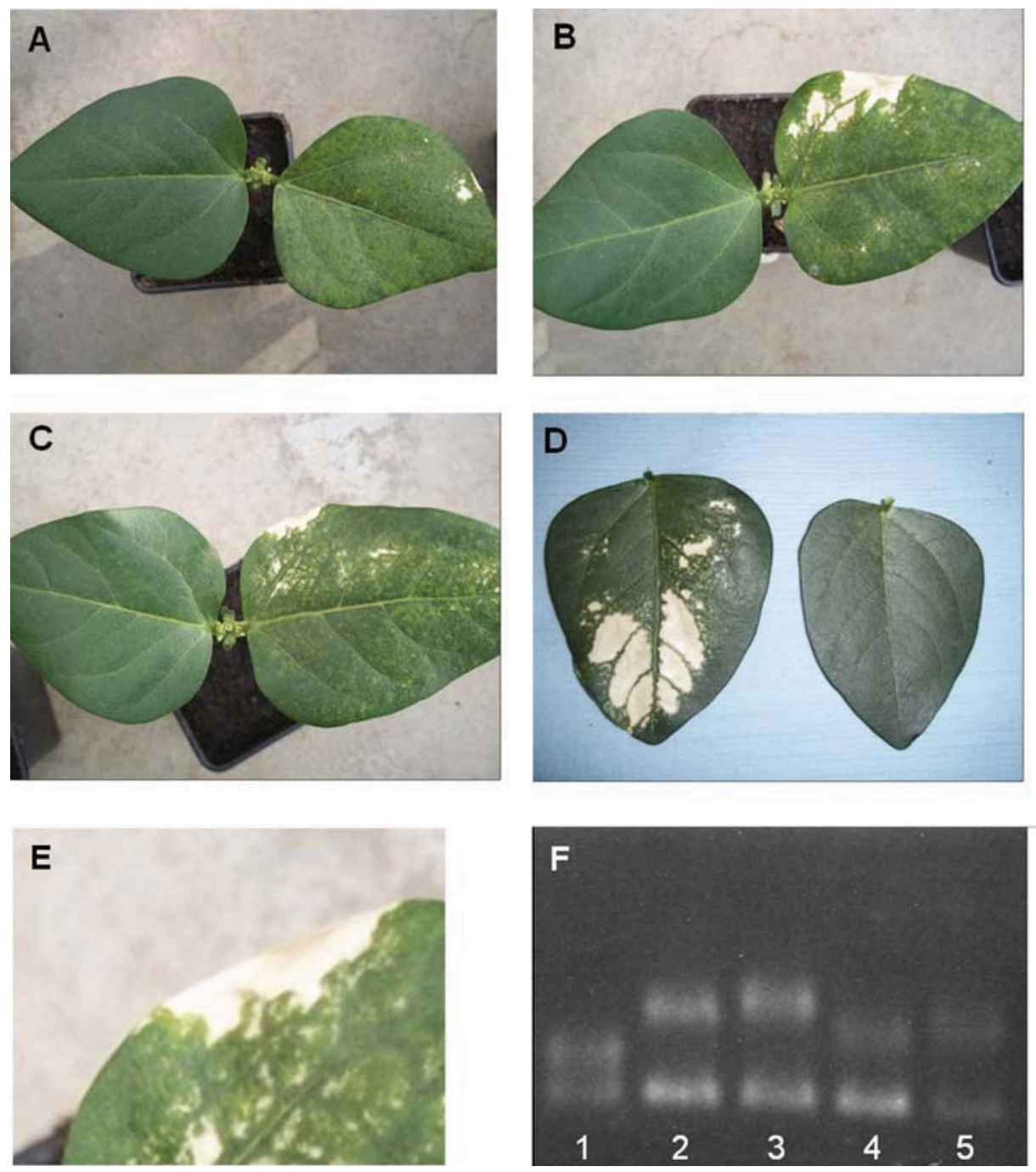

Figure 3. (A-D) Optical images of symptomatic V. unguiculata plants viewed after infection with aqueous dispersions of WT-CPMV (A), Cat-CPMV (B) or [Cat-CPMV][S], or solvent-free liquid [Cat-CPMV][S] (D). In each case, pairs of leaves either treated or untreated with the infective agents are shown. (E) High magnification image of leaf surface following infection by aqueous [Cat-CPMV][S] conjugate $\left(50 \mu \mathrm{L}\right.$ of a $\left.0.1 \mathrm{mg} \mathrm{mL}^{-1}\right)$ showing pale green patches indicative of infection, as well as white patches corresponding to dead leaf tissue. (F) Agarose gel electrophoresis profiles of WT-CPMV (lane 1), WT-CPMV from Cat-CPMV infection (lane 2), WT-CPMV from aqueous [Cat-CPMV][S] infection (lane 3), WT-CPMV from control infection with WT-CPMV (lane 4), and WT-CPMV from infection with [Cat-CPMV][S] in the melt state (lane 5).

We attribute the high plant infectivity of the [Cat-CPMV] [S] construct to a combination of factors associated with effective intercellular transport of the virions due to the structural integrity and retention of the viral RNA, as well as efficient viral replication facilitated by dissociation of the electrostatically attached coronal layer under conditions of increased ionic strength. Infection by plant viruses generally requires mechanical damage to the cell wall, for example by a vector organism feeding on the plant, ${ }^{[31]}$ which provides direct entry of the virions into the cell. Although the exact mechanism for the subsequent release and translation of viral RNA in CPMV is unknown, proposed mechanisms for other plant viruses suggest that the RNA uncoating and translation processes occur simultaneously due to structural transitions or partial dissociation of viral capsids at specific sites due to cation- or membrane-binding within the cell followed by ribosomal translation of the partially exposed
RNA strands. In the case of cowpea chlorotic mottle virus, these structural changes occur at the pentameric capsomer that forms a vertex in the icosahedral virus capsid, ${ }^{[32]}$ suggesting that pores present at similar positions in CPMV could be sites for partial exposure of the genetic material, particularly in view of the absence of extensive interactions between ends of the individual protein subunits ${ }^{[26]}$

We also investigated the potential for exploiting the virus melts and soft solids in the development of non-aqueous processing routes. Previous reports have indicated that capsid-like proteins such as ferritin can be transferred into dichloromethane through the surface attachment of hydrophobic chains, ${ }^{[33,34]}$ and significantly, a similar approach has been recently developed to prepare water-free, organic solutions of bacteriophage $\mathrm{MS}^{[35]}$ and tobacco mosaic virus (TMV). ${ }^{[36]}$ We therefore tested the solubility of the lyophilized [Cat-CPMV][S] materials in a variety of organic 

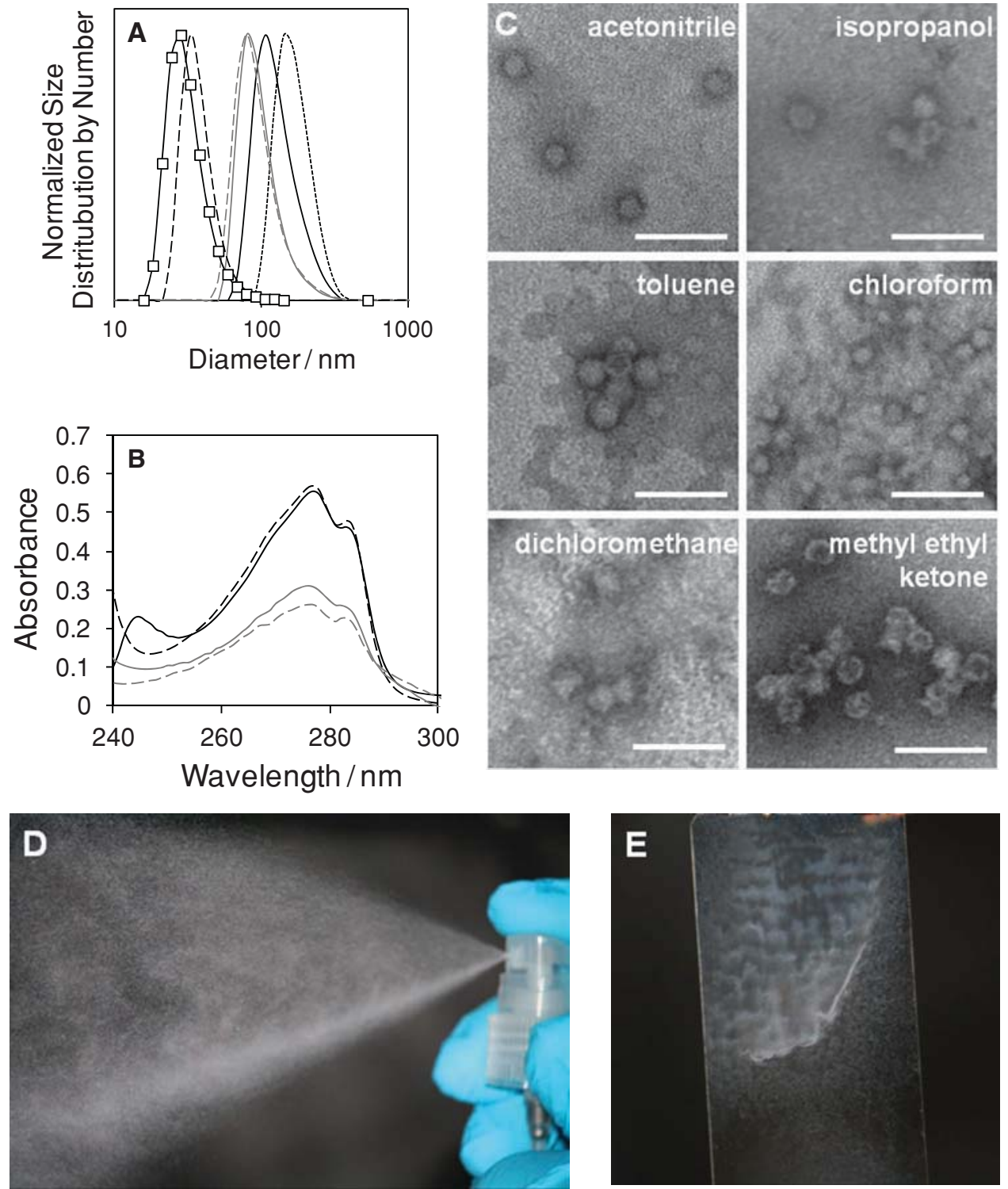

Figure 4. (A) DLS plots of size distribution by number of a dispersion of [Cat-CPMV][S] in acetonitrile (grey dashed line), isopropanol (grey solid line), toluene $(\square)$, chloroform (black solid line), dichloromethane (black dashed line) and methyl ethyl ketone (dotted line). (B) Corresponding UV-Vis spectra of [Cat-CPMV][S] in acetonitrile (grey dashed line), isopropanol (grey solid line), chloroform (black solid line), dichloromethane (black dashed line). (C) Transmission electron micrographs of [Cat-CPMV][S] dissolved in various organic solvents showing intact virus particles in each case. Samples were stained with $1 \%$ phosphotungstic acid prior to imaging. Scale bars $=100 \mathrm{~nm}$. (D) Optical image showing aerosol spray of [Cat-CPMV][S] dissolved in toluene at a concentration of $1 \mathrm{mg} \mathrm{mL}^{-1}$. (E) Film of [Cat-CPMV][S] produced on a glass slide after aerosol droplet deposition and subsequent evaporation of the chloroform solvent.

solvents. Number-averaged size distributions obtained by DLS indicated that the lyophilized water-free [Cat-CPMV][S] nanoconstruct was soluble in range of organic solvents (Figure 4A). The DLS plots for toluene and dichloromethane show discretely dispersed particles of mean hydrodynamic diameter less than $40 \mathrm{~nm}$, whereas aggregated clusters of $c a .100,130$, or $170 \mathrm{~nm}$ in diameter were observed in acetonitrile and isopropanol, chloroform or methyl ethyl ketone, respectively, which indicated that the nature of the solvent had significant effects on the dispersion of the nanoconstructs. UV-Vis spectra showed the characteristic absorbance peak of $\mathbf{S}$ at $275 \mathrm{~nm}$ (Figure 4B), and TEM images confirmed the presence of intact virions in each of the organic solvents (Figure 4C). Given the propensity for dissolution in low boiling point organic solvents, we developed proof-of-concept studies to demonstrate that aerosol delivery of the surfaceengineered plant virus was feasible. For this, the lyophilized [Cat-CPMV][S] material was dissolved in toluene or chloroform at a concentration of $1 \mathrm{mg} \mathrm{mL}^{-1}$, and the resulting solution sprayed using a commercially available mist sprayer to produce micronsized aerosol droplets (Figure 4D). Thin films of the [Cat-CPMV] $[\mathrm{S}]$ virions were readily produced after solvent evaporation of the aerosols by spraying onto a variety of substrates (Figure 4E). 
Finally, we extended the general principles of our synthetic strategy towards the nanoscale engineering of capsid surfaces by successfully preparing a solvent-free liquid of TMV particles, which are considerably larger $(18 \mathrm{~nm} \times 300 \mathrm{~nm})$ and more anisotropic than the CPMV virions. Significantly, TEM micrographs of the solvent-free [Cat-TMV][S] construct after re-dissolution in water showed intact cylindrical virions with a polypeptide helical superstructure and nanoscale internal channel (Supporting Information Figure S7A). DSC profiles of the solvent-free [Cat-TMV][S] construct showed distinct crystallization and melting temperatures at $-27^{\circ} \mathrm{C}$ and $28{ }^{\circ} \mathrm{C}$, respectively (Supporting Information Figure S7B), and AUC and DLS profiles confirmed that both the [Cat-TMV] and [CatTMV][S] conjugates were discrete nanoconstructs and devoid of unbound S molecules (Supporting Information Figure S7C,D).

In conclusion, a synthetic strategy for the nanoscale engineering of capsid surfaces that enables a new class of nanostructured biohybrid materials to be prepared by rational design has been described. The polymer-surfactant/virus constructs are produced as highly concentrated solvent-free viscoelastic liquids or soft solids that can be readily stored, transported and used as infective agents. Furthermore, the surface-engineered virions can be re-dissolved in aqueous media or dissolved in a range of organic solvents, and easily sprayed as aerosols for potential delivery and application on synthetic or biological substrates, suggesting that such materials could find diverse uses as functional hybrid components in many areas of bioinspired nanotechnology.

\section{Acknowledgements}

We thank the EPSRC (Cross-disciplinary Interfaces Program) and ERC (Advanced Grant Scheme) for financial support for AWP and SM, respectively. SM is indebted to the Radcliffe Institute for Advanced Study, Harvard University, USA, for the award of an International Fellowship during 2011-12. NM and AJP are grateful for financial support from the EPSRC and University of Bristol, UK respectively. We also thank the BBSRC, UK for funding of a Core Strategic Grant to the John Innes Centre (JEB and DJE), Alaa A. A. Aljabali (John Innes Centre) for technical assistance. Antje Völkel, Rose Rosenberg and Dirk Haffke for their skillfull assistance with AUC, James Lewis for macro-photography, and David Williams and Alex P. S. Brogan for generating space-filled models of CPMV particles and useful discussions.

[1] K. T. Nam, D.-W. Kim, P. J. Yoo, C.-Y. Chiang, N. Meethong, P. T. Hammond, Y.-M. Chiang, A. M. Belcher, Science 2006, 312, 885.

[2] D. J. Evans, J. Mater. Chem. 2008, 18, 3746.

[3] T. Douglas, M. Young, Nature 1998, 393, 152.
[4] E. Dujardin, C. Peet, G. Stubbs, J. N. Culver, S. Mann, Nano Lett. 2003, 3, 413

[5] S. E. Aniagyei, C. DuFort, C. C. Kao, B. Dragnea, J. Mater. Chem. 2008, 18, 3763.

[6] L. Loo, R. H. Guenther, S. A. Lommel, S. Franzen, J. Am. Chem. Soc. 2007, 129, 11111.

[7] C. E. Fowler, W. Shenton, G. Stubbs, S. Mann, Adv. Mater. 2001, 13, 1266.

[8] Y. J. Lee, H. Yi, W.-J. Kim, K. Kang, D. S. Yun, M. S. Strano, G. Ceder, A. M. Belcher, Science 2009, 324, 1051.

[9] Q. Wang, T. Lin, L. Tang, J. E. Johnson, M. G. Finn, Angew. Chem. Int. Ed. 2002, 41, 459.

[10] M. C. Canizares, L. Nicholson, G. P. Lomonossoff, Immuno. Cell Biol. 2005, 83, 263.

[11] Y. P. Ren, S. M. Wong, L. Y. Lim, Pharm. Res. 2010, 27, 2509.

[12] G. Destito, R. Yeh, C. S. Rae, M. G. Finn, M. Manchester, Chem. Biol. 2007, 14, 1152.

[13] A. S. Blum, C. M. Soto, C. D. Wilson, T. L. Brower, S. K. Pollack, T. L. Schull, A. Chatterji, T. W. Lin, J. E. Johnson, C. Amsinck, P. Franzon, R. Shashidhar, B. R. Ratna, Small 2005, 1, 702.

[14] N. F. Steinmetz, S. N. Shah, J. E. Barclay, G. Rallapalli, G. P. Lomonossoff, D. J. Evans, Small 2009, 5, 813.

[15] N. F. Steinmetz, G. Calder, G. P. Lomonossoff, D. J. Evans, Langmuir 2006, 22, 10032

[16] R. Usha, J. B. Rohll, V. E. Spall, M. Shanks, A. J. Maule, J. E. Johnson, G. P. Lomonossoff, Virology 1993, 197, 366.

[17] C. Porta, V. E. Spall, J. Loveland, J. E. Johnson, P. J. Barker, G. P. Lomonossoff, Virology 1994, 202, 949.

[18] A. A. A. Aljabali, F. Sainsbury, G. P. Lomonossoff, D. J. Evans, Small 2010, 6, 818 .

[19] K. Saunders, F. Sainsbury, G. P. Lomonossoff, Virology 2009, 393, 329.

[20] Y. J. Min, M. Akbulut, K. Kristiansen, Y. Golan, J. Israelachvili, Nat. Mater. 2008, 7, 527.

[21] A. W. Perriman, S. Mann, ACS Nano 2011, 5, 6085.

[22] A. W. Perriman, H. Coelfen, R. W. Hughes, C. L. Barrie, S. Mann, Angew. Chem. Int. Ed. 2009, 48, 6242.

[23] A. W. Perriman, A. P. S. Brogan, H. Coelfen, N. Tsoureas, G. R. Owen, S. Mann, Nat. Chem. 2010, 2, 622.

[24] N. F. Steinmetz, G. P. Lomonossoff, D. J. Evans, Langmuir 2006, 22, 3488.

[25] A. A. A. Aljabali, J. E. Barclay, J. N. Butt, G. P. Lomonossoff, D. J. Evans, Dalton Trans. 2010, 39, 7569.

[26] T. W. Lin, Z. G. Chen, R. Usha, C. V. Stauffacher, J. B. Dai, T. Schmidt, J. E. Johnson, Virology 1999, 265, 20.

[27] L. Pauling, J. Am. Chem. Soc. 1945, 67, 555.

[28] J. A. Rupley, E. Gratton, G. Careri, Trends Biochem. Sci. 1983, 8, 18.

[29] I. W. Hamley, Introduction to Soft Matter: Polymers, Colloids, Amphiphiles and Liquid Crystals, Wiley, Chichester, 2000.

[30] M. Banyay, M. Sarkar, A. Gräslund, Biophys. Chem. 2003, 104, 477.

[31] R. Hull, Matthews' Plant Virology, 4th ed., Academic Press, New York 2002.

[32] F. G. Albert, J. M. Fox, M. J. Young, J. Virology 1997, 71, 4296.

[33] K. K. W. Wong, N. T. Whilton, T. Douglas, S. Mann, H. Colfen, Chem. Commun. 1998, 1621.

[34] K. K. W. Wong, H. Coelfen, N. T. Whilton, T. Douglas, S. Mann, J. Inorg. Biochem. 1999, 76, 187.

[35] H. R. Johnson, J. M. Hooker, M. B. Francis, D. S. Clark, Biotechnol. and Bioeng. 2007, 97, 224.

[36] P. G. Holder, D. T. Finley, N. Stephanopoulos, R. Walton, D. S. Clark, M. B. Francis, Langmuir 2010, 26, 17383. 